\title{
The Impact of Diabetes Education Modality and Diabetes Type on Psychosocial Outcomes in Young Adults with Diabetes
}

\author{
Heather Blume ${ }^{1, *}$, SeAnne Safaii ${ }^{2}$, Samantha Ramsay ${ }^{1}$, Martha Raidl ${ }^{2}$ \\ ${ }^{1}$ College of Agriculture and Life Sciences, University of Idaho, Moscow, 83844, Idaho, United States \\ ${ }^{2}$ College of Agriculture and Life Sciences, University of Idaho, Boise, 83702, Idaho, United States \\ *Corresponding Author: hblume011@gmail.com
}

Copyright (C) 2013 Horizon Research Publishing All rights reserved.

\begin{abstract}
A four lesson diabetes curriculum and two discussion sessions, focusing on diabetes self-management (diet, medication, physical activity) were delivered via two educational modalities: 1) Traditional Face-to-Face (FTF) Classes and 2) Virtual Diabetes Education (VDE) Classes administered in the Second Life ${ }^{\circledR}$ virtual world. The purpose of the study was to 1) describe the psychosocial outcomes in young adults with type 1 and type 2 diabetes and 2) determine whether two types of educational modalities (FTF or VDE) impacted the psychosocial outcomes of young adults with diabetes. A convenience sample of young adults with diabetes $(\mathrm{N}=81), 18$ to 28 years-old, completed pre and post psychosocial surveys that measured levels of depression, self-efficacy, and social support. Multivariate and univariate analyses of variance identified differences in psychosocial outcomes. Overall, $29.9 \%$ of young adult participants reported depressive symptoms at the beginning of the study, and those with type 2 diabetes had higher rates of depressive symptoms (44.4\%) than participants with type 1 diabetes $(26.6 \%)$. Individuals with type 1 diabetes had a higher level of self-efficacy $(\mathrm{p}=.000)$ and more social support $(\mathrm{p}=.03)$ than their peers with type 2 diabetes. No statistically significant differences were apparent among type 1 and type 2 diabetes groups at post study measures. Face-to-Face participants had significantly greater $(p=.02)$ gains in levels of diabetes self-efficacy. Participants in the FTF condition improved mean diabetes self-efficacy scores and participants in the VDE condition decreased mean diabetes self-efficacy scores. Session attendance was higher in the FTF condition $(x=3.72, S D=2.23)$ than among participants assigned to the VDE condition $(x=1.79, \mathrm{SD}=1.82)$. Education and support programs should address differences in psychosocial outcomes based on both diabetes type and educational modality.
\end{abstract}

Keywords Diabetes, Young Adults, Psychosocial, Virtual

\section{Introduction}

According to the 2011 National Diabetes Fact Sheet, a total of 25.8 million children and adults in the United States have diabetes [1], and $23 \%$ of adolescents have prediabetes or diabetes [2]. Individuals with diabetes often struggle with managing blood glucose levels, balancing food intake, engaging in physical activity, and dealing with the psychological stress of living with the chronic condition [3]. Young adults have additional challenges to manage the disease because they also are seeking to assert their independence while dealing with issues such as finances, establishing relationships, exploring career options, and transitioning their healthcare from pediatric care to adult care [4]. These challenges make it difficult for young adults to find the proper support they need to manage their condition [5].

Whether diagnosed with type 1 or type 2 diabetes, young adults with diabetes must address the emotional demands of living with a chronic illness [6]. Three psychosocial variables- depression, self-efficacy, and social supportaffect their ability to manage their diabetes care. Psychological distress in young adults with type 1 diabetes is prevalent, including depression, and emotional or behavior problems [7]. Males with type 2 diabetes typically exhibit higher rates of depressive symptoms than males with type 1 diabetes [8] and individuals with diabetes and comorbid depression are more likely to experience hyperglycemia and increased risk for complications [9]. Diabetes related distress has a strong effect on diabetes quality of life and general well-being [10].

The frequency of diabetes-related distress can be influenced by an individual's self-efficacy, or perception of his or her ability to succeed in a particular situation as well as overcome the difficulties inherent in that situation [11]. An individual's self-efficacy plays a role in how goals, tasks, and challenges are approached. In addition to self-efficacy, social support can mediate distress [12] and play a role in adherence to diabetes self-management [4]. 
Psychosocial variables constitute an important part of the experience of living with diabetes and can impact adherence to diabetes management, levels of glycemic control, occurrence of complications, and quality of life [13]. The demands of managing diabetes [14] combined with the challenges inherent in transitioning to adulthood [4] prompt the need for diabetes education programs specific to the young adult population.

A variety of educational models exist, but new trends toward virtual technology education programs are beginning to occur. Professionals in both the fields of diabetes and technology recommend using virtual worlds for diabetes management training [15], for weight loss [16], and to encourage people to make healthier food choices[15]. The Second Life ${ }^{\circledR}$ virtual world is the largest virtual landscape [17] and may be an effective means of conducting medical education [18]. The Second Life $\mathbb{R}$ world provides an opportunity for participants to interact with each other and diabetes educators using avatars. As online and virtual education modalities increase in prevalence, educators need to better understand their efficacy [19] and impact on young adults with diabetes.

Limited evidence is available regarding how to address psychosocial issues related to diabetes management in young adults, and research is especially sparse concerning young adults with type 2 diabetes [20]. Additionally, there is lack of research regarding educational modalities for young adults with diabetes [20], particularly virtual diabetes education modalities [21]. Therefore, the purpose of the study was 1) to describe the psychosocial outcomes in young adults with type 1 and type 2 diabetes and 2) to determine whether two types of educational modalities (FTF or VDE) impacted the psychosocial outcomes of young adults with diabetes. Psychosocial outcomes considered include level of depression, self-efficacy and social support.

\section{Materials and Methods}

\subsection{Participants}

Young adults $(\mathrm{N}=97)$ were recruited from diabetes education centers located in two states, Idaho and Washington, from January 2011 to August 2011. Eligibility criteria included being 18 to 28 years-old and being diagnosed with type 1 or type 2 diabetes.

\subsection{Study Design}

Participants provided informed consent and were compensated for their participation. Participants were assigned to either the FTF condition or the VDE condition based on preference, distance from the education site, and computer experience. Prior to assignment, participants were paired based on initial HbA1c levels, gender, and computer experience. Subjects who lacked appropriate computer hardware or virtual media experience were assigned to the
FTF condition and the paired partner was assigned to the VDE condition. Partners that exhibited equal computer aptitude and equipment were assigned to their condition via a virtual coin toss. Reassignments were not made and the final condition assignment pre-study was 52 participants in the FTF condition, and 45 participants in the VDE condition. Attrition was defined as drop-out from one or more measurement sessions. A group of 30 participants chose to abstain from the free educational sessions. This resulted in a de facto, no-intervention control group. Accounting for the Control condition and the decision of 16 individuals to drop out, the final condition assignment was 35 in FTF, 24 in VDE, and 22 in the Control group. Responses to questionnaires on diabetes related self-efficacy, depressive symptoms, and perceived social support were collected at pre and post study. Open-ended questions were also asked regarding course content and recommendations.

\subsection{Six Session Diabetes Program}

Participants were encouraged to participate in four diabetes-related classes and two discussion sessions at no cost. The curriculum was delivered over six months and feedback regarding the two different educational modalities was gathered post-study. The sessions were delivered via a FTF setting or a VDE setting created in the Second Life ${ }^{\circledR}$ virtual world. The study session curriculum was developed using information from focus groups [22]. The following topics were included: 1) diabetes types, the physiology of diabetes, medication, and testing blood glucose; 2) managing hyper- and hypoglycemia, the effects of alcohol and recreational drugs on blood glucose, and eating out; 3) exercise, new technologies, cooking, and weight management; 4) dietary supplements, summer BBQs, journaling, sick day guidelines, birth control, and pregnancy; 5) group discussion one on food and exercise; 6) group discussion two on emotional ups and downs of life with diabetes.

\subsection{Psychosocial Measures}

Three validated surveys were used to collect psychosocial data. The Center for Epidemiologic Studies Depression (CES-D) Scale measured the level of depressive symptoms among participants. The Diabetes Self-Efficacy Scale was used to measure level of self-efficacy related to diabetes. The Social Provisions Scale measured level of social support participants received.

\subsubsection{Center for Epidemiologic Studies Depression (CES-D) Scale}

The CES-D is a 20 question survey that determines level of depressive symptoms (e.g. depressed mood, feelings of worthlessness, feelings of hopelessness, loss of appetite, poor concentration, and sleep disturbance). The CES-D score can range from 0 to 60 , with higher scores indicating more severe depressive symptoms [23]. Elevated depressive 
symptoms were defined by a CES-D score of 16 or higher, consistent with mild to moderate depression or dysthymia [9].

\subsubsection{Diabetes Self-Efficacy Scale}

The Diabetes Self-Efficacy Scale is an eight question survey developed by the Stanford Patient Education Research Center [24]. Participants were asked to rate each item on a scale from 1 to 10 based on their level of confidence to complete diabetes related self-management tasks. The scores were reported as a mean response, with higher scores indicating higher self-efficacy [25].

\subsubsection{Social Provisions Scale}

The Social Provisions Scale is a 24 question survey that measures participants' need and perceived social support. The scale examines the extent to which an individual's social relationships provide different elements of social support [26]. Mean scores at or below 2.5 indicate that the participant did not agree that they had social support [27].

\subsection{Statistical Analyses}

The reliability of the psychosocial scales at pre and post study was tested using Cronbach's $\alpha$, with acceptability indicated by a score of .70 or higher [28]. At pre-study measurements the CES-D, Diabetes Self-Efficacy, and Social Provisions scales all had acceptable reliability. Results for post-study Diabetes Self-Efficacy should be interpreted with caution, as the reliability of .68 may indicate questionable reliability [28].

The data was tested for normality and differences between psychosocial responses based on education setting (FTF, VDE, or Control), diabetes type, and gender were analyzed using a multivariate analysis of variance (MANOVA). The Hotellings Trace statistic was used to determine level of significance. Univariate (ANOVA) tests were used to analyze any subsequent differences among means. Differences in mean responses from pre to post study were calculated by subtracting the pre-study responses from the post-study responses. Responses to the three psychosocial scales constituted the dependent variables while education modality, diabetes type, and gender were treated as independent factors in the MANOVA. All tests were two-tailed and used a significance level of $\mathrm{p}<.05$. All analysis were conducted using SPSS version 20.0 [29].

\section{Results}

\subsection{Demographic Characteristics}

Of the original 97 participants, 81 individuals provided pre and post psychosocial measurements. A greater number of women participated in the study than men and the majority of participants had type 1 diabetes (versus type 2 see Table 1.)
Participants with type 2 diabetes had a significantly lower $[F(1,79)=19.68, M S E=29.21, p=.000]$ mean duration of diabetes, approximately five years, than those with type 1 diabetes, approximately 12 years.

\subsection{Psychosocial Outcomes}

At pre-study, 29.9\% ( $\mathrm{n}=29)$ of all participants were categorized as depressed $(\geq 16$ on the CES-D). At pre-study, $26.6 \%(\mathrm{n}=21)$ of individuals with type 1 diabetes reported depressive symptoms ( $\geq 16$ on the CES-D) and $44.4 \%(\mathrm{n}=8)$ of individuals with type 2 reported depressive symptoms ( $\geq 16$ on the CES-D). There was no significant change in rates of depressive symptoms by post study.

Table 1. Participants Gender, Age, and Diabetes Duration by Diabetes Type

\begin{tabular}{|c|c|c|c|}
\hline Characteristic & Type 1 & Type 2 & Total \\
\hline Gender & $\mathrm{n}(\%)$ & $\mathrm{n}(\%)$ & $\mathrm{n}(\%)$ \\
\hline Male & $26(32.1)$ & $5(6.2)$ & $31(38.3)$ \\
\hline Female & $39(48.1)$ & $11(13.6)$ & $50(61.7)$ \\
\hline Total & $65(80.2)$ & $16(19.8)$ & $81(100)$ \\
\hline $\begin{array}{c}\text { Age (years) } \\
\text { (years) }\end{array}$ & $23.4 \pm 0.35$ & $25.3 \pm 0.82$ & $23.8 \pm 0.34$ \\
\hline $\begin{array}{c}\text { Diabetes Duration } \\
\text { (yesE }\end{array}$ & $11.8 \pm 0.71 *$ & $5.1 \pm 0.88 *$ & $10.5 \pm 0.67$ \\
\hline
\end{tabular}

*Significant difference in mean diabetes duration $(\mathrm{p}=.000)$ by diabetes type

Participants with type 1 diabetes reported significantly different psychosocial outcomes than participants with type 2 diabetes [Hotellings Trace $=0.19, \mathrm{~F}(3,77)=4.87, \mathrm{p}=.004]$.

No significant differences in psychosocial measures among groups based on gender, diabetes type, or education setting were identified at post-study.

Pre-study results in Table 2 show that individuals with type 1 diabetes reported significantly higher levels of Diabetes Self-Efficacy $[\mathrm{F}(1,79)=14.13, \mathrm{MSE}=2.05, \mathrm{p}=.000]$ and Social Provisions $[\mathrm{F}(1,79)=4.92, \mathrm{MSE}=0.12, \mathrm{p}=.03]$ than individuals with type 2 diabetes. There was no significant difference in CES-D scores between the two groups.

Post-study results in Table 2 report no significant difference between type 1 and type 2 participants in Diabetes Self-Efficacy, Social Provisions, and CES-D scores. The results in Table 3 show that type 2 participants increased their Diabetes Self-Efficacy and Social Provisions and decreased their level of depression, as evidenced by the drop in CES-D scores.

Individuals with type 1 diabetes reported higher pre-study Diabetes Self-Efficacy scores than individuals with type 2 diabetes. The mean Social Provisions Score was also higher for individuals with type 1 diabetes than for 
individuals with type 2 diabetes. Mean CES-D scores for type 2 participants were higher than mean CES-D scores for type 1 participants, indicating higher rates of depression in participants with type 2 diabetes.

At post-study, participants with type 1 diabetes continued to have higher Diabetes Self-Efficacy scores than participants with type 2 diabetes and higher Social Provisions scores. Participants with type 1 diabetes reported lower mean CES-D scores than participants with type 2 diabetes at post-study.

As indicated in Table 3, mean Diabetes Self-Efficacy scores had a greater increase for participants with type 2 diabetes than participants with type 1 diabetes. Improvements in Social Provisions scores were greater for participants with type 2 diabetes than for participants with type 1 diabetes. Mean CES-D scores decreased more for participants with type 2 diabetes than for participants with type 1 diabetes.

\subsection{Diabetes Education Modality and Curriculum}

Changes in psychosocial outcomes over the course of the study were analyzed using a reduced model MANOVA with education setting as a factor. The reduced model demonstrated a possible difference in psychosocial changes over the course of the study among groups based on education setting [Hotellings Trace $=0.16, \mathrm{~F}(6,150)=2.05$, $\mathrm{p}=.06]$. A univariate analysis of variance (ANOVA) model showed changes in Diabetes Self-Efficacy from pre to post scores were significantly different $[\mathrm{F}(2,78)=3.92$, $\mathrm{MSE}=1.83, \mathrm{p}=.02]$ among groups based on education setting.

Participants assigned to the FTF condition attended significantly more $[\mathrm{F}(1,79)=17.96, \mathrm{MSE}=4.18, \mathrm{p}=.000]$ sessions on average $[x=3.72, S E=0.34]$ than participants assigned to the VDE condition $[\mathrm{x}=1.79, \mathrm{SE}=0.29]$. This equates to an attendance rate of approximately $60 \%$ in the FTF condition and $30 \%$ in the VDE condition. By the end of the study, $66.7 \%(n=52)$ of participants stated they would choose the FTF educational setting over VDE format if they participated again. Barriers to attendance included computer difficulties, dislike of the virtual condition, and schedule conflicts with work and school..

Table 2. Pre and Post-Study Psychosocial Outcomes by Diabetes Type

\begin{tabular}{|c|c|c|c|c|c|c|c|}
\hline \multirow{2}{*}{} & \multicolumn{2}{|c|}{$\begin{array}{c}\text { Type 1 } \\
(\mathrm{n}=65)\end{array}$} & \multicolumn{2}{c|}{$\begin{array}{c}\text { Type } 2 \\
(\mathrm{n}=16)\end{array}$} & \multicolumn{2}{c|}{$\begin{array}{c}\text { Total } \\
(\mathrm{n}=81)\end{array}$} \\
\hline \multirow{2}{*}{ Psychosocial Scale } & $\mathrm{M} \pm \mathrm{SE}$ & $95 \% \mathrm{CI}$ & $\mathrm{M} \pm \mathrm{SE}$ & $95 \% \mathrm{CI}$ & $\mathrm{M} \pm \mathrm{SE}$ & $95 \%$ CI \\
\hline \multirow{2}{*}{$\begin{array}{c}\text { Diabetes } \\
\text { Self-Efficacy }\end{array}$} & Pre & $7.92 \pm 0.16^{* *}$ & {$[7.59,8.24]$} & $6.41 \pm 0.47 * *$ & {$[5.41,7.42]$} & $7.62 \pm 0.17$ & {$[7.28,7.96]$} \\
\cline { 2 - 9 } & Post & $7.98 \pm 0.13$ & {$[7.72,8.24]$} & $7.38 \pm 0.26$ & {$[6.86,7.90]$} & $7.86 \pm 0.12$ & {$[7.62,8.10]$} \\
\hline \multirow{2}{*}{$\begin{array}{c}\text { Social } \\
\text { Provisions }\end{array}$} & Pre & $3.59 \pm 0.04^{*}$ & {$[3.52,3.67]$} & $3.38 \pm 0.12^{*}$ & {$[3.11,3.65]$} & $3.55 \pm 0.04$ & {$[3.47,3.63]$} \\
\cline { 2 - 9 } & Post & $3.64 \pm 0.04$ & {$[3.56,3.72]$} & $3.51 \pm 0.09$ & {$[3.33,3.69]$} & $3.61 \pm 0.03$ & {$[3.55,3.67]$} \\
\hline \multirow{2}{*}{ CES-D } & Pre & $12.03 \pm 1.23$ & {$[9.58,14.49]$} & $15.94 \pm 2.58$ & {$[10.46,21.43]$} & $12.80 \pm 1.12$ & {$[10.58,15.02]$} \\
\cline { 2 - 8 } & Post & $11.85 \pm 1.36$ & {$[9.13,14.57]$} & $15.06 \pm 2.64$ & {$[9.78,20.34]$} & $12.48 \pm 1.21$ & {$[10.06,14.90]$} \\
\hline
\end{tabular}

*Statistically significant $(\mathrm{p} \leq .05)$ difference between type 1 and type 2 diabetes mean Social Provision scores **Statistically significant $(\mathrm{p} \leq .001)$ difference between type 1 and type 2 mean Diabetes Self-Efficacy scores $\dagger$ Self-Efficacy range from 0 (very low self-efficacy) to 10 (very high self-efficacy).

$\dagger \dagger \mathrm{A}$ score of $\leq 2.5$ indicates that respondents feel like they are lacking social support

Table 3. Differences between Pre and Post Psychosocial Outcomes by Diabetes Type

\begin{tabular}{|c|c|c|c|c|c|c|}
\hline & \multicolumn{2}{|c|}{$\begin{array}{c}\text { Type 1 } \\
(\mathrm{n}=65)\end{array}$} & \multicolumn{2}{c|}{$\begin{array}{c}\text { Type 2 } \\
(\mathrm{n}=16)\end{array}$} & \multicolumn{2}{c|}{$\begin{array}{c}\text { Total } \\
(\mathrm{n}=81)\end{array}$} \\
\hline $\begin{array}{c}\text { Psychosocial } \\
\text { Scale }\end{array}$ & $\mathrm{M} \pm \mathrm{SE}$ & $95 \% \mathrm{CI}$ & $\mathrm{M} \pm \mathrm{SE}$ & $95 \% \mathrm{CI}$ & $\mathrm{M} \pm \mathrm{SE}$ & $95 \%$ CI \\
\hline $\begin{array}{c}\text { Diabetes } \\
\text { Self-Efficacy }\end{array}$ & $0.06 \pm 0.16$ & {$[-0.26,0.37]$} & $0.97 \pm 0.42$ & {$[0.07,1.87]$} & $0.24 \pm 0.16$ & {$[-0.07,-0.55]$} \\
\hline $\begin{array}{c}\text { Social } \\
\text { Provisions }\end{array}$ & $0.05 \pm 0.03$ & {$[-0.02,0.11]$} & $0.13 \pm 0.08$ & {$[-0.05,0.31]$} & $0.06 \pm 0.03$ & {$[0.00,0.12]$} \\
\hline CES-D & $-0.19 \pm 1.41$ & {$[-3.00,2.63]$} & $-0.88 \pm 1.91$ & {$[-4.94,3.19]$} & $-0.32 \pm 1.19$ & {$[-2.68,2.04]$} \\
\hline
\end{tabular}


Table 4. Mean Responses to Pre and Post Measures of Psychosocial Health Based on Education Setting

\begin{tabular}{|c|c|c|c|c|c|c|}
\hline & Pre & & Post & & \multicolumn{2}{|c|}{ Post-Pre Difference } \\
\hline & $\mathrm{M} \pm \mathrm{SE}$ & $\mathrm{CI}$ & $\mathrm{M} \pm \mathrm{SE}$ & CI & $\mathrm{M} \pm \mathrm{SE}$ & CI \\
\hline \multicolumn{7}{|c|}{ DiabetesSelf-Efficacy } \\
\hline $\begin{array}{c}\text { FTF } \\
(\mathrm{n}=35)\end{array}$ & $7.44 \pm 0.29$ & {$[6.85,8.04]$} & $8.04 \pm 0.17$ & {$[7.71,8.38]$} & $0.60 \pm 0.30^{*}$ & {$[-.01,1.21]$} \\
\hline $\begin{array}{c}\mathrm{VDE} \\
(\mathrm{n}=24)\end{array}$ & $7.99 \pm 0.26$ & {$[7.45,8.53]$} & $7.60 \pm 0.22$ & {$[7.14,8.06]$} & $-0.39 \pm 0.19^{*}$ & {$[-.78,0.00]$} \\
\hline $\begin{array}{c}\text { Control } \\
(\mathrm{n}=22)\end{array}$ & $7.50 \pm 0.32$ & {$[6.84,8.16]$} & $7.85 \pm 0.26$ & {$[7.31,8.39]$} & $0.35 \pm 0.19^{*}$ & {$[-.06, .75]$} \\
\hline $\begin{array}{c}\text { Total } \\
(\mathrm{n}=81)\end{array}$ & $7.62 \pm 0.17$ & {$[7.28,7.96]$} & $7.86 \pm 0.12$ & {$[7.62,8.10]$} & $0.24 \pm 0.16$ & {$[-.07, .55]$} \\
\hline \multicolumn{7}{|c|}{ Social Provisions } \\
\hline $\begin{array}{c}\text { FTF } \\
(\mathrm{n}=35)\end{array}$ & $3.50 \pm 0.05$ & {$[3.40,3.60]$} & $3.59 \pm 0.05$ & {$[3.49,3.68]$} & $.09 \pm 0.04$ & {$[0.00, .17]$} \\
\hline $\begin{array}{c}\mathrm{VDE} \\
(\mathrm{n}=24)\end{array}$ & $3.56 \pm 0.07$ & {$[3.41,3.71]$} & $3.61 \pm 0.07$ & {$[3.47,3.75]$} & $.05 \pm 0.05$ & {$[-.05, .14]$} \\
\hline $\begin{array}{l}\text { Control } \\
(\mathrm{n}=22)\end{array}$ & $3.62 \pm 0.09$ & {$[3.43,3.81]$} & $3.66 \pm 0.07$ & {$[3.52,3.81]$} & $.05 \pm 0.08$ & {$[-.11, .20]$} \\
\hline $\begin{array}{c}\text { Total } \\
(\mathrm{n}=81)\end{array}$ & $3.55 \pm .04$ & {$[3.47,3.63]$} & $3.61 \pm .03$ & {$[3.55,3.68]$} & $.06 \pm .03$ & {$[0.00, .12]$} \\
\hline \multicolumn{7}{|c|}{ CES-D } \\
\hline $\begin{array}{c}\text { FTF } \\
(\mathrm{n}=35)\end{array}$ & $13.89 \pm 1.93$ & {$[9.96,17.81]$} & $11.49 \pm 1.72$ & {$[7.98,14.99]$} & $-2.40 \pm 2.00$ & {$[-6.47,1.66]$} \\
\hline $\begin{array}{c}\mathrm{VDE} \\
(\mathrm{n}=24)\end{array}$ & $12.88 \pm 1.94$ & {$[8.85,16.90]$} & $12.38 \pm 1.98$ & {$[8.28,16.47]$} & $-.50 \pm 1.38$ & {$[-3.35,2.35]$} \\
\hline $\begin{array}{l}\text { Control } \\
(\mathrm{n}=22)\end{array}$ & $11.00 \pm 1.76$ & {$[7.34,14.66]$} & $14.18 \pm 2.85$ & {$[8.26,20.11]$} & $3.18 \pm 2.50$ & {$[-2.02,8.39]$} \\
\hline $\begin{array}{c}\text { Total } \\
(\mathrm{n}=81)\end{array}$ & $12.80 \pm 1.12$ & {$[10.58,15.02]$} & $12.48 \pm 1.21$ & {$[10.07,14.90]$} & $-.32 \pm 1.19$ & {$[-2.68,2.04]$} \\
\hline
\end{tabular}

* Difference $(p=.06)$ between mean Diabetes Self-Efficacy scores based on education setting

Table 4 shows that, compared to the VDE group, participants in the FTF group had a greater increase in Diabetes Self-Efficacy and decrease in CES-D scores. For both groups, the Social Provisions scores remained virtually unchanged.

Participants assigned to the FTF condition reported the greatest gain in Diabetes Self-Efficacy, from a mean score of 7.44 to 8.04 , while individuals in the VDE condition decreased slightly in Diabetes Self-Efficacy, from a mean score of 7.99 to 7.60 , and Control scores increased slightly, from 7.50 to 7.85 (Table 4). Mean pre-study Social Provisions scores were similar in the FTF, VDE, and Control groups and post-study scores stayed the same. Though not statistically different among groups, CES-D scores decreased for FTF participants from 13.89 to 11.49 and for the VDE group from 12.88 to 12.38 (Table 4). Means CES-D scores for the Control group increased from 11.00 to 14.18 (Table 4).

Almost one-third of FTF participants (31.4\%) listed discussion two: "Emotional Ups and Downs of Life with Diabetes" as the topic they would most like to see covered in an advanced follow-up class. Only $4.3 \%$ of the VDE participants desired a follow-up on emotions and coping. When asked to evaluate the two discussion sessions, participants in both the FTF and VDE conditions stated that being able to share experiences and talk with their peers was beneficial. Face-to-Face participant recommendations for improving the classes included more interaction with peers and the desire for longer class times. Virtual participant responses focused more on improving the technical aspect of the VDE classes, but also included comments on feeling more motivated after "talking about everything" and feeling less alone.

\section{Discussion}

The present study provided information regarding 1) psychosocial outcomes in the young adult population with diabetes and 2) the impact of education setting on psychosocial outcomes. While previous research has discussed the psychosocial issues of young adults with type 1 diabetes $[7,20,30]$ the current study provided information regarding the experience of young adults with both type 1 and type 2 diabetes. In particular, young adults with type 2 diabetes have lower levels of diabetes self-efficacy and less social support than their peers with type 1 diabetes.

Study participants indicated a desire for more social support from peers, which can have a positive impact on self-care behaviors [4], improve quality of life, and increase self-efficacy [31].

The current study provided new information regarding rates of depression in young adults with diabetes. Previous research demonstrates that the prevalence of depression in 
adults with diabetes ranges from $21.3 \%$ to $31.1 \%$. Though some researchers have presented information regarding rates of depression in adults with diabetes [32], young adults aged 18 to 28 were not included. A greater amount of research has demonstrated the rate of depression in type 1 diabetes than type 2 diabetes. The SEARCH for Diabetes in Youth study reported rates of depression for youth aged 10 to 18 , but did not report on rates of depression in young adults [8]. Thirty-five percent of young adults with type 1 diabetes had depressive symptoms ( $\geq 16$ on the CES-D) [7], and the current study demonstrated that $26.6 \%$ of young adults with type 1 diabetes had depressive symptoms. Study participants with type 2 diabetes demonstrated higher rates of depression (44.4\%) than peers with type 1 diabetes, which is consistent with comparisons of depression rates in adolescents with diabetes [8]. The prevalence of depression did not change significantly over the course of the study and advanced sessions on emotional coping were requested by FTF participants.

The pre-study mean Diabetes Self-Efficacy score was high in the present study, and the mean Diabetes Self-Efficacy score for young adults with type 2 diabetes $(\mathrm{x}=6.41)$ was comparable to that found in other studies [33]. Diabetes Self-Efficacy scores differed for individuals with type 1 and type 2 diabetes pre-study.

When considering the educational modality, participants in the FTF condition had greater improvements in Diabetes Self-Efficacy and attended more classes. Additionally, at the end of the study, participants were more likely to prefer the FTF educational settings. Challenges associated with implementing a virtual technology modality have been identified, such as ease of use and computer literacy[19,34]. Feedback from participants indicates that improvements will need to be made in VDE technologies and implementation before it is acceptable to young adults.

Though there is the potential for virtual technologies to address psychosocial concerns [35] and foster desirable health behaviors [19], it was not demonstrated in this study. Further research is needed as study duration was short and attendance was low. However, virtual worlds provide the opportunity to reach individuals who cannot attend class due to geographical distances and may appeal to young adults who are accustomed to engaging with virtual settings in gaming scenarios. To our knowledge there is no prior research regarding diabetes education conducted in a virtual format, therefore this study serves as a preliminary exploration of virtual technology modalities. Further research is needed to continue examining the efficacy of virtual reality as a diabetes education setting.

A small number of participants and poor attendance of educational sessions were limitations to this study. Level of attendance could have influenced the psychosocial measures as lack of attendance resulted in fewer opportunities for activities, discussion, and networking to improve upon the measured psychosocial skills. Lack of attendance among young adults has previously been cited as a concern for diabetes education programs [36]. Additional limitations included a small number of participants with type 2 diabetes $(\mathrm{n}=16)$ and a self-selected control group $(n=22)$. The small number of participants in the VDE condition $(n=24)$ may have limited the ability to detect differences between educational settings. Therefore, it is recommended that additional research be conducted on educational programs that incorporate psychosocial interventions and support.

\section{Conclusion}

The current study provides information regarding the psychosocial experience of managing diabetes in young adulthood and addresses the lack of information regarding virtual modalities and psychosocial outcomes. This study shows that levels of self-efficacy and social support differ by diabetes type and that individuals with type 2 diabetes have a higher rate of depression than individuals with type 1 diabetes.

Participants in the VDE condition had decreased levels of Diabetes Self-Efficacy, while participants in the FTF group saw an improvement in Diabetes Self-Efficacy. Levels of social support and depression remained unchanged, regardless of education setting. Attendance was higher among participants in the FTF condition than in the VDE group. Participants listed computer difficulties, dislike of the virtual condition, and schedule conflicts with work and school as barriers to attendance in the VDE condition. As this is one of the few studies conducted on the use of virtual education for diabetes [19], the discovery of outcomes and barriers to participation can be used to guide future studies. As shown in this study, virtual modalities do not address psychosocial needs in young adults with diabetes.

\section{Acknowledgements}

We are very grateful to the Virtual Technology and Design department for their collaboration in creating the virtual diabetes education center and to Humphrey's Diabetes Center and the Rockwood Clinic for their partnership conducting the diabetes classes.

\section{REFERENCES}

[1] Centers for Disease Control and Prevention, National diabetes fact sheet: National estimates and general information on diabetes and prediabetes in the United States, 2011, Atlanta, GA, 2011.

[2] A. May, E. Kuklina, P. Yoon, Prevalence of cardiovascular disease risk factors among US adolescents, 1999-2008, Pediatrics, Vol. 129, No. 6, 1035-1041, 2012.

[3] M. Abolfotouh, M. Kamal, M. El-Bourgy, S. Mohamed, Quality of life and glycemic control in adolescents with type 1 
diabetes and the impact of an education intervention, International Journal of General Medicine, Vol. 4, 141-52, 2011.

[4] J. Weissberg-Benchell, H. Wolpert, B. J. Anderson, Transitioning from pediatric to adult care: A new approach to the post-adolescent young person with type 1 diabetes, Diabetes Care, Vol. 30, No. 10, 2441-2446, 2007.

[5] N. Van Walleghem, C. MacDonald, H. Dean, Evaluation of a systems navigator model for transition from pediatric to adult care for young adults with type 1 diabetes, Diabetes Care, Vol. 31, No. 8, 1529-1530, 2008.

[6] M. Botello-Harbaum, T. Nansel, D. L. Haynie, R. J. Iannotti, B. Simons-Morton, Responsive parenting is associated with improved type 1 diabetes-related quality of life, Child: Care, Health and Development, Vol. 34, No. 5, 675-81, 2008.

[7] A. L. Hislop, P. G. Fegan, M. J. Schlaeppi, M. Duck, B. B. Yeap, Prevalence and associations of psychological distress in young adults with type 1 diabetes, Diabetic Medicine: A Journal of the British Diabetic Association, Vol. 25, No. 1, 91-6, 2008

[8] J. Lawrence, D. Standiford, B. Loots, G. Klingensmith, D. Williams, A. Ruggiero, A. Liese, R. Bell, B. Waitzfelder, R. McKeown, Prevalence and correlates of depressed mood among youth with diabetes: The SEARCH for Diabetes in Youth study, Pediatrics, Vol. 117, No. 4, 1348-58, 2006.

[9] [9] R. Anderson, K. Freedland, R. Clouse, P. Lustman, The prevalence of comorbid depression in adults with diabetes: A meta-analysis, Diabetes Care, Vol. 24, No. 6, 1069-1078, 2001.

[10] [10] J. Malik, H. Koot, Explaining the adjustment of adolescents with type 1 diabetes role of diabetes-specific and psychosocial factors, Diabetes Care, Vol. 32, No. 5, 774-779, 2009.

[11] A. Bandura, Self-efficacy: Toward a unifying theory of behavioral change, Psychological Review, Vol. 84, No. 2, 191-215, 1977.

[12] J. Malik, H. Koot, Assessing diabetes support in adolescents: Factor structure of the modified Diabetes Social Support Questionnaire (DSSQ-Friends), Diabetic Medicine, Vol. 29, No. 8, e232-40, 2012.

[13] P. Trief, J. Teresi, R. Izquierdo, P. Morin, R. Goland, L. Filed, J. Eimicke, R. Brittain, J. Starren, S. Shea, R. Weinstock, Psychosocial outcomes of telemedicine case management for elderly patients, Diabetes Care, Vol. 30, No. 5, 1266-1268, 2007.

[14] B. Anderson, Living with depression and type 1 or type 2 diabetes in late adolescence and young adulthood: Lessons from research, Diabetes Spectrum, Vol. 23, No. 1, 32-37, 2010.

[15] B. Ruppert, New directions in virtual environments and gaming to address obesity and diabetes: Industry perspective, Journal of Diabetes Science and Technology, Vol. 5, No. 2, 277-82, 2011.

[16] M. J. Coons, M. Roehrig, B. Spring, The potential of virtual reality technologies to improve adherence to weight loss behaviors, Journal of Diabetes Science and Technology, Vol. 5, No. 2, 340-4, 2011.
[17] Educause Learning Initiative, 7 things you should know about Second Life, Online available from http://www.educause.edu/library/resources/7-things-you-sho uld-know-about-second-life, 2008.

[18] J. Wiecha, R. Heyden, E. Sternthal, M. Merialdi, Learning in a virtual world: Experience with using second life for medical education, Journal of Medical Internet Research, Vol. 12, No. 1, e1, 2010.

[19] A. Ershow, C. Peterson, W. Riley, A. S. Rizzo, B. Wansink, Virtual reality technologies for research and education in obesity and diabetes: Research needs and opportunities, Journal of Diabetes Science and Technology, Vol. 5, No. 2, 212-24, 2011.

[20] A. Peters, L. Laffel, The American Diabetes Association Transitions Working Group, Diabetes care for emerging adults: Recommendations for transition from pediatric to adult diabetes care systems, Diabetes Care, Vol. 34, No. 11, 2477-85, 2011.

[21] B. Ruppert, New directions in the use of virtual reality for food shopping: Marketing and education perspectives, Journal of Diabetes Science and Technology, Vol. 5, No. 2, $315-8,2011$

[22] S. Safaii, M. Raidl, S. Ramsay, L. Galbraith, Barriers to Management in 18-28 year olds with Type I and Type II Diabetes, American Dietetic Association Food and Nutrition Conference and Exhibition, 2011.

[23] L. Radloff, The CES-D Scale: A self-report depression scale for research in the general population, Applied Psychological Measurement, Vol. 1, No. 3, 385-401, 1977.

[24] Stanford School of Medicine, Research instruments developed, adapted or used by the Stanford Patient Education Research Center, Patient Education in the Department of Medicine, 2012. Online available from http://patienteducation.stanford.edu/research/

[25] Stanford Patient Education Research Center, Self-efficacy for diabetes. Stanford Patient Education Research Center, Palo Alto, 1-2, 2013.

[26] C. Cutrona, D. Russel, Social Provisions Scale, in Measures for Assessment of Functioning and Outcomes in Longitudinal Research on Child Abuse Volume 2: Middle Childhood, University of North Carolina, United States, 472-479, 2003.

[27] B. Mallinckrodt, J. M. Armer, P. P. Heppner, A threshold model of social support, adjustment, and distress after breast cancer treatment, Journal of Counseling Psychology, Vol. 59, No. 1, 150-60, 2012.

[28] J. Gliem, R. Gliem, Calculating, interpreting, and reporting Cronbach's Alpha reliability coefficient for Likert-type scales, Midwest Research to Practice Conference in Adult, Continuing, and Community Education, 82-88, 2003.

[29] IBM Corp, IBM SPSS Statistics for Windows. IMB Corp, Armonk, NY, 2011.

[30] V. Wilson, Students' experiences of managing type 1 diabetes, Paediatric Nursing, Vol. 22, No. 10, 25-8, 2010.

[31] J. Markowitz, L. Laffel, Transitions in care: Support group for young adults with type 1 diabetes, Diabetic Medicine: A Journal of the British Diabetic Association, Vol. 29, No. 4, 522-5, 2012. 
[32] J. Kaholokula, S. Haynes, A. Grandinetti, H. Chang, Biological, psychosocial, and sociodemographic variables associated with depressive symptoms in persons with type 2 diabetes, Journal of Behavioral Medicine, Vol. 26, No. 5, 435-458, 2003.

[33] P. McGowan, The efficacy of diabetes patient education and self-management education in type 2 diabetes, Canadian Journal of Diabetes, Vol. 35, No. 1, 46-53, 2011.

[34] M. Fleming, D. Olsen, H. Stathes, L. Boteler, P. Grossberg, J. Pfeifer, S. Schiro, J. Banning, S. Skochelak, Virtual reality skills training for health care professionals in alcohol screening and brief intervention, Journal of the American
Board of Family Medicine, Vol. 22, No. 4, 387-98, 2009.

[35] T. Parsons, A. Rizzo, Affective outcomes of virtual reality exposure therapy for anxiety and specific phobias: A meta-analysis, Journal of Behavior Therapy and Experimental Psychiatry, Vol. 39, No. 3, 250-61, 2008.

[36] R. Snow, N. Fulop, Understanding issues associated with attending a young adult diabetes clinic: A case study, Diabetic Medicine: A Journal of the British Diabetic Association, Vol. 29, No. 2, 257-9, 2012. 